\title{
Identification of JAK/STAT signalling components by genome-wide RNA interference
}

\author{
Patrick Müller ${ }^{1}$, David Kuttenkeuler ${ }^{2}$, Viola Gesellchen ${ }^{2}$, Martin P. Zeidler ${ }^{1}$ \& Michael Boutros ${ }^{2}$
}

Signalling pathways mediating the transduction of information between cells are essential for development, cellular differentiation and homeostasis ${ }^{1}$. Their dysregulation is also frequently associated with human malignancies. The Janus tyrosine kinase/ signal transducer and activator of transcription (JAK/STAT) pathway represents one such signalling cascade whose evolutionarily conserved roles include cell proliferation and haematopoiesis ${ }^{2}$. Here we describe a systematic genome-wide survey for genes required for JAK/STAT pathway activity. Analysis of 20,026 RNA interference (RNAi)-induced phenotypes in cultured Drosophila melanogaster haemocyte-like cells identified interacting genes encoding 4 known and 86 previously uncharacterized proteins. Subsequently, cell-based epistasis experiments were used to classify these proteins on the basis of their interaction with known components of the signalling cascade. In addition to multiple human disease gene homologues, we have found the tyrosine phosphatase Ptp61F and the Drosophila homologue of BRWD3, a bromo-domain-containing protein disrupted in leukaemia ${ }^{3}$. Moreover, in vivo analysis demonstrates that disrupted $d B R W D 3$ and overexpressed Ptp61F function as suppressors of leukaemialike blood cell tumours. This screen represents a comprehensive identification of novel loci required for JAK/STAT signalling and provides molecular insights into an important pathway relevant for human cancer. Human homologues of identified pathway modifiers may constitute targets for therapeutic interventions.

Developmental genetic screens in Drosophila have identified multiple JAK/STAT pathway components on the basis of their segmentation phenotype $e^{4-6}$, and subsequent analysis of the pathway has characterized evolutionarily conserved roles during immune responses, haematopoiesis and cellular proliferation ${ }^{7-10}$. The JAK/ STAT signalling cascade in Drosophila comprises the extracellular ligand Unpaired $(\mathrm{Upd})^{5}$, a transmembrane receptor with homology to the interleukin 6 (IL-6) receptor family termed Domeless (Dome) $)^{11}$, a single Janus tyrosine kinase (JAK) called Hopscotch $(\mathrm{Hop})^{4}$ and the Stat92E transcription factor ${ }^{6,12}$ (Fig. 1a). Known regulators of JAK/STAT signalling, including a family of SOCS-like genes $^{13}, d P I A S / S u(v a r) 2-10$ (ref. 14) and STAM (ref. 15), are functionally conserved and were identified based on their homology to components originally characterized in mammalian cell culture studies ${ }^{2}$. Although successful in identifying the pathway members Upd, Dome, Hop and Stat92E, it is probable that forward genetic approaches have missed components, possibly due to non-saturating mutagenesis, genetic redundancy or phenotypic pleiotropy.

In order to identify novel pathway components and circumvent limitations of classical genetic screens, we have undertaken a genome-wide RNAi screen, a powerful technique for the identification of new components of diverse cellular pathways ${ }^{16-19}$. To this end, we devised a quantitative assay for JAK/STAT signalling activity in cultured Drosophila cells by multimerizing a STAT92E-binding site from the Draf promotor ${ }^{20}$ to generate the $6 \times 2 \times$ DrafLuc firefly luciferase reporter. Given the role for JAK/STAT signalling in haematopoiesis ${ }^{9}$, we used Drosophila haemocyte-like $\mathrm{Kc}_{167}$ cells because of their endogenous ability to respond to pathway activation (Fig. 1b). On transfection of the $6 \times 2 \times$ DrafLuc reporter and a plasmid to
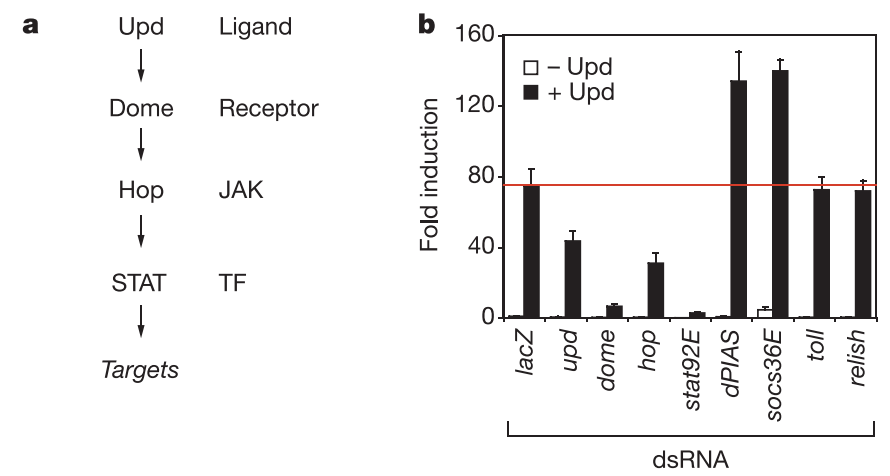

c Batch transfection of $\mathrm{Kc}_{167}$ cells with pathway reporter and inducer
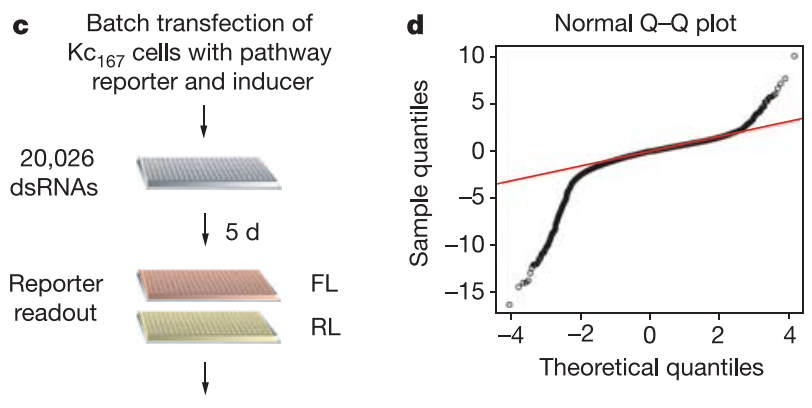

Computational analysis secondary assays

Figure 1 | Genome-wide RNAi screen for JAK/STAT signalling factors. a, Schematic representation of the Drosophila JAK/STAT signalling pathway. TF indicates transcription factor. $\mathbf{b}$, Knock down of known JAK/STAT components leads to loss of pathway induction by Upd, whereas knock down of lac $Z$, toll and relish show no effect. The red line indicates an approximately 70-fold reporter induction relative to negative control dsRNA. Error bars represent standard deviations of six experiments. c, Schematic diagram of screening approach. A total of 20,026 dsRNAs were screened in duplicate in 384-well plates before computational analysis and re-testing. FL indicates firefly luciferase; RL indicates Renilla luciferase. d, Q-Q plot of normally distributed quantiles against actual pathway screening result quantiles. A fit to a normal distribution is represented by the red line. Tails of positively and negatively interacting dsRNAs at each extreme with a $z$-score threshold of $>2$ and $<-2$ represent RNAi experiments with significant phenotypes. 
constitutively express the ligand $\mathrm{Upd}$, a robust induction of reporter gene activity was observed (Fig. 1b). We examined whether depletion of known pathway components by $\mathrm{RNAi}^{21}$ modifies JAK/STAT signalling activity in $\mathrm{Kc}_{167}$ cells. We assessed the effect of doublestranded (ds)RNAs targeting the mRNA of the genes dome, stat92E and hop, as well as dsRNAs directed against the negative regulators socs $36 E$ and dPIAS. As shown in Fig. 1b, knock down of JAK/STAT components results in significant changes in reporter activity, whereas reporter activity in uninduced cells remains at low levels (Fig. 1b).
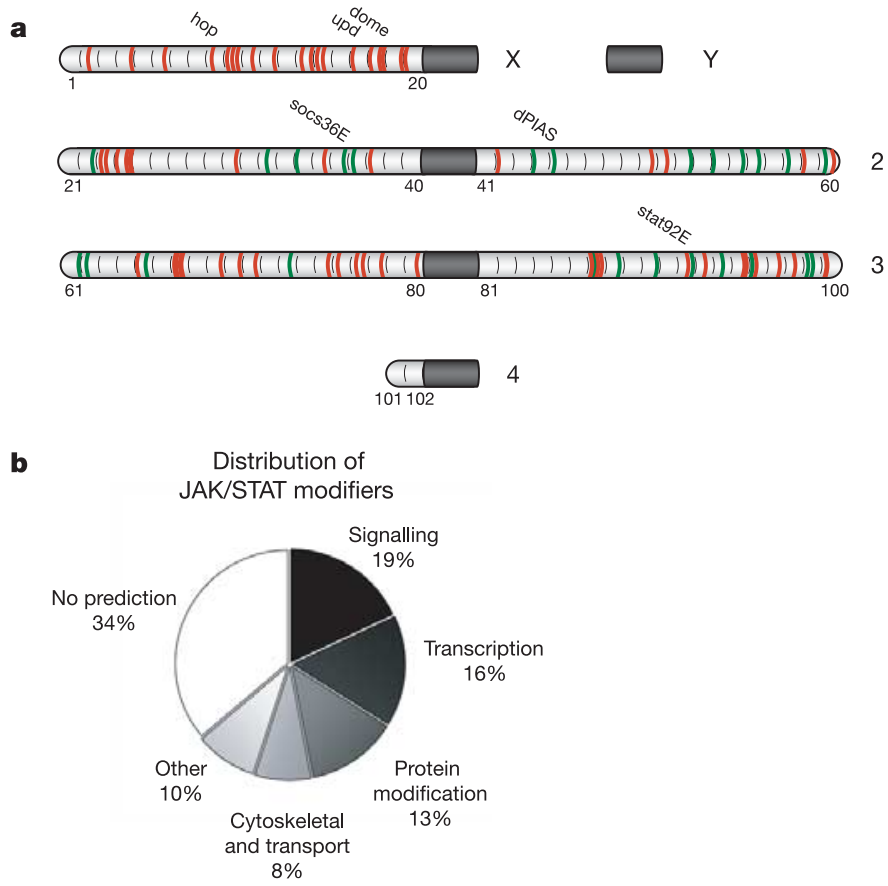

c

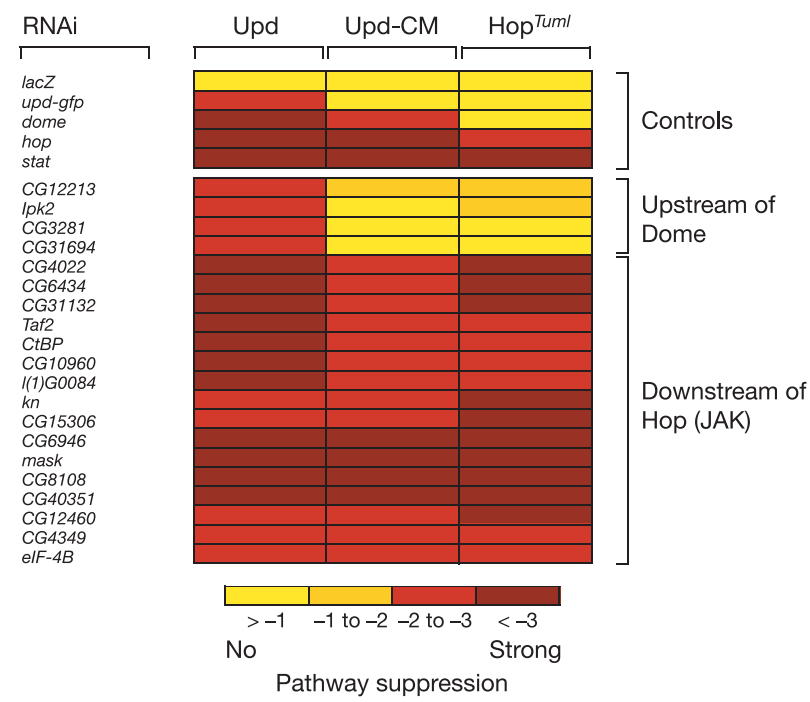

Figure 2 Analysis of JAK/STAT activity modulators. a, Schematic representation of positive (red) and negative (green) regulator loci distributed within the Drosophila genome. An interactive version of this panel is available at http://www.dkfz.de/signalling/jak-pathway/. b, Distribution of predicted gene functions. c, Epistasis analysis of the indicated positive pathway regulators showing interactions graded from none (yellow squares) to strong (red squares). Results shown have been obtained in two independent replicate experiments. Abbreviations used are: ectopic expression of $u p d$ (Upd); Upd-conditioned medium (Upd-CM); and expression of a constitutively active JAK-allele $\left(\mathrm{Hop}^{\text {Tuml }}\right)$ (ref. 26). Colour coding of $z$-scores is shown.
We then set out to systematically identify genes required for JAK/ STAT signalling by generating a library of 20,026 dsRNAs targeting $\sim 91 \%$ of the predicted transcripts in the Drosophila genome (see Supplementary Information). Using this library we performed duplicate genome-wide screens as outlined in Fig. 1c and Supplementary Fig. S1. After computational analysis to identify candidate pathway modifiers (Fig. 1d; see also Supplementary Information), dsRNAs were resynthesized and assayed with an independent reporter, derived from the promoter of the pathway target gene $\operatorname{socs} 36 E^{13}$, to exclude reporter-specific artefacts. These approaches confirmed the identification of 67 dsRNAs that decrease pathway activity (putative positive regulators) and 24 dsRNAs that

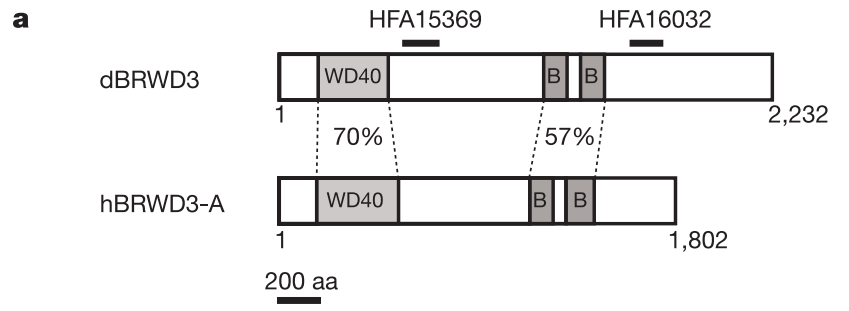

b

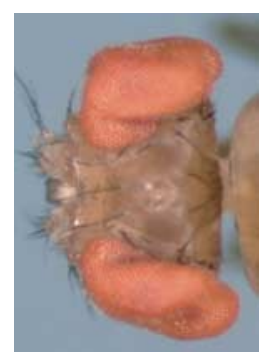

GMR-upd $\Delta 31 /+$ $+/+$

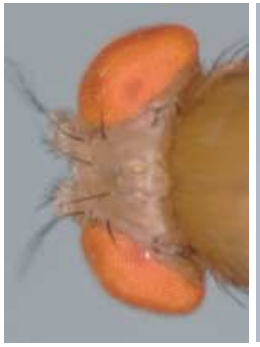

GMR-upd $\Delta 3^{1} /+$; STAT92E $06346 /+$

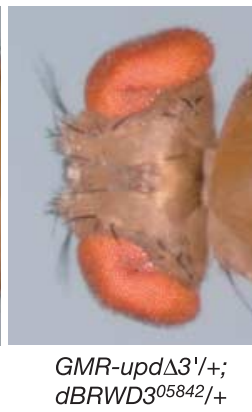

c

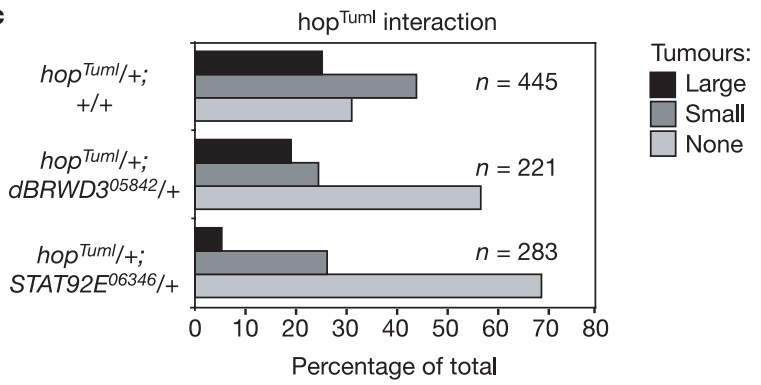

d

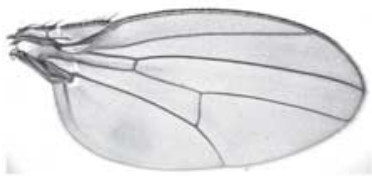

Wild type

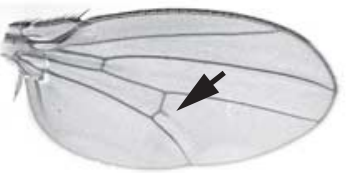

dBRWD3 310
Figure 3 | dBRWD3 functions as a JAK/STAT pathway component. a, Domain structure and sequence similarity of Drosophila and human BRWD3 proteins. Percentages show the similarity in the amino acid sequence, and regions targeted by two independent dsRNAs independently recovered in the screen are shown. b, Adult Drosophila heads heterozygous for the GMR-upd $\Delta 3^{\prime}$ transgene crossed to wild type (left panel), stat92E (middle panel) and $d B R W D 3$ (right panel) mutants. Note the reduction in eye overgrowth after removal of pathway components. c, The size and frequency of $h o p^{T u m l}$-induced tumour formation is significantly decreased in stat $92 E$ and $d B R W D 3$ heterozygous backgrounds. d, Compared to adult wild-type wings (left), ectopic wing vein material (arrow) is present in homozygous $d B R W D 3^{\triangle 10}$ mutant flies (a putative hypomorphic allele, right), a phenotype reminiscent of the stat $92 E^{H J}$ mutant $^{12}$. 
increase pathway activity (putative negative regulators) (see also Supplementary Materials and Table 1), targeting a total of 90 predicted genes. Although most modifiers are distributed throughout the genome (Fig. 2a; see also Supplementary Table 7), the X chromosome appears to be devoid of negative regulators, a finding that may be linked to the role of the pathway in X:autosome ratio detection during Drosophila sex determination ${ }^{22}$.

Based on Interpro and gene ontology (GO) annotations, pathway modifiers were classified according to their predicted functions (see Supplementary Information). Signalling factors, enzymes mediating post-translational protein modifications and transcription factors cumulatively represent $48 \%$ of the genes identified (Fig. 2b). Examples include CG11501 encoding a putatively secreted, negative regulator of JAK/STAT signalling previously demonstrated to be a pathway target gene ${ }^{8}$, enok/CG11290 encoding an acetyl-transferase, and the tumour suppressor protein 101/CG9712 gene, which encodes a ubiquitin conjugating enzyme. The molecular role of these genes in the regulation of JAK/STAT signalling remains to be determined. Seventy-four per cent of the identified loci possess human homologues ( $E$-values $<10^{-10}$, compared to $62 \%$ for the whole proteome), of which $39 \%$ have been implicated in human disease (see Supplementary Tables 5 and 6).

A genetic technique to characterize signalling molecules is the determination of their epistatic relationship with respect to defined pathway components. We therefore performed cell-based epistatic assays to determine the pathway response to expression of upd, Upd conditioned medium or expression of the constitutively active JAK allele $h o p^{\text {Tuml }}$ (refs 5, 23) while simultaneously targeting a subset of positive regulators. In this way, dsRNA-inactivated genes required upstream in the pathway can be characterized on the basis of their rescue by pathway activation further downstream (Fig. 2c). For example, although depletion of the $\gamma$-interferon-related protein CG31694 results in downregulation of signalling stimulated by expression of $u p d$, activation by Upd-conditioned medium or $h o p^{\text {Tuml }}$ is unaffected (Fig. 2c). This suggests that CG31694 is required for the production and/or activity of the Upd ligand. Conversely, loss of pathway activity resulting from the knock down of CG31132 cannot be rescued by any form of pathway stimulus, implying a function downstream of JAK (Fig. 2c). Although this analysis suggests a role for multiple genes upstream of Dome, this classification is based on the lack of interaction observed under differing experimental conditions and the molecular basis of these results remains to be established.

In order to confirm the function of candidate genes in vivo, we tested examples of both positive and negative regulators of the JAK/ STAT signalling pathway. One positive regulator mentioned above is CG31132, which encodes a 2,232-amino-acid WD40- and bromodomain-containing protein homologous to human BRWD3 (Fig. 3a; see also Supplementary Information). In the screen, a strong reduction of pathway activity was observed for two independent dsRNAs that target different regions of the transcript (Fig. 3a). BRWD3 is a functionally uncharacterized locus recently identified at the break point of $\mathrm{t}(\mathrm{X} ; 11)(\mathrm{q} 13 ; \mathrm{q} 23)$ translocations derived from multiple B cell chronic lymphocytic leukaemia (B-CLL) patients ${ }^{3}$.

A previously uncharacterized mutagenic $P$ element inserted in the fourth intron of $C G 31132$ (hereafter termed $d B R W D 3^{05842}$ ) has been generated by the Drosophila genome project ${ }^{24}$ and its remobilization indicates that the transposon insertion is responsible for late embryonic lethality (see Supplementary Information). We therefore tested for genetic interactions between $A B R W D 3$ and JAK/STAT signalling by crossing the $d B R W D 3^{05842}$ allele to GMR-upd $\Delta 3^{\prime}$ (ref. 25). The $G M R-u p d \Delta 3^{\prime}$ transgene ectopically misexpresses upd during eye development, resulting in cellular overproliferation and an enlarged adult eye (Fig. 3b, left panel). Furthermore, removal of one copy of stat92E significantly suppresses eye overgrowth (Fig. 3b, middle panel) due to a reduction in the potency of JAK/STAT signalling ${ }^{25}$. Removal of a single copy of $d B R W D 3$ was also able to suppress the $G M R$-upd $\Delta 3^{\prime}$ phenotype (Fig. 3b, right panel) as expected for a a

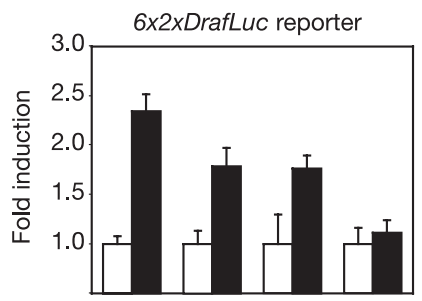

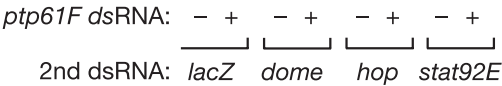

b

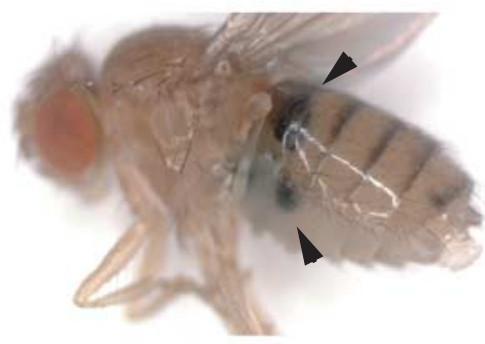

hop ${ }^{T u m} /$ cg-Gal4 / +

Figure 4 | Ptp61F is a tumour suppressor in vivo. a, Epistasis analysis of $p t p 61 F$ dsRNA in cell culture indicates that it acts downstream of Hop and upstream or parallel to STAT92E. b, Haemocyte-specific misexpression of ptp $61 \mathrm{~F}$ can protect hop ${ }^{\text {Tuml }}$ mutants from melanotic tumour formation. Compare large black tumours in controls (arrowheads, left) with small tumours present in a $p$ tp $61 F$-expressing individual (right). c, Quantitative
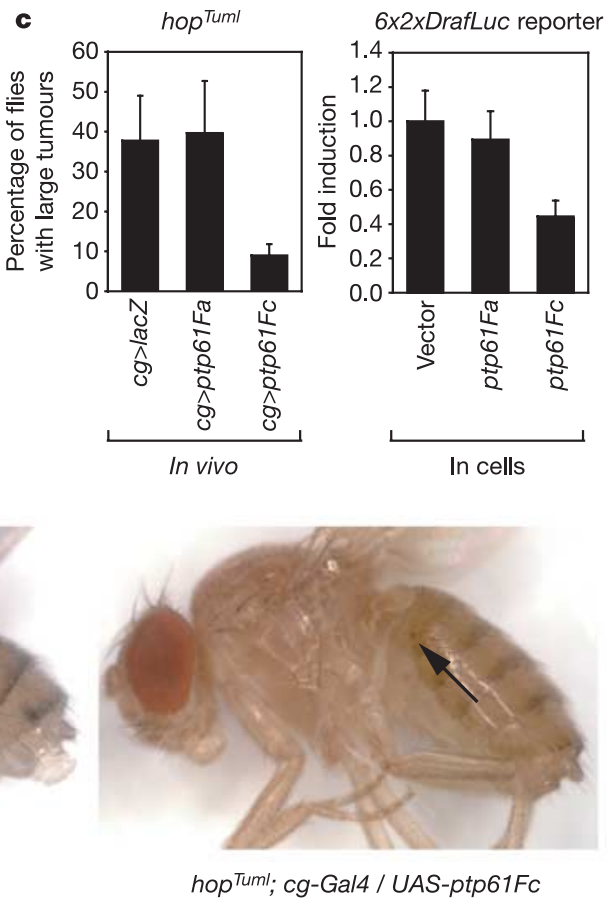

analysis of large tumour formation in hop ${ }^{\text {Tuml }}$ mutants expressing cytoplasmic Ptp61Fa and nuclear Ptp61Fc, showing specificity of rescue for the nuclear isoform (left), an effect that is mirrored by overexpression of the same isoforms in tissue-culture-based reporter assays (right). Error bars represent standard deviations of three or four independently tested transgenic lines or eight parallel cell culture experiments. 
positive regulator of JAK/STAT signalling. A chromosomal deficiency removing the region has previously been independently identified as a suppressor of GMR-upd $\Delta 3^{\prime}$ (ref. 25).

One phenotypic consequence of constitutive JAK/STAT activation caused by the gain-of-function JAK allele hop ${ }^{\text {Tuml }}$ is the overproliferation of haemocytes and the frequent formation of melanotic tumours, a phenotype described as a Drosophila model for leukae$\mathrm{mia}^{23,26}$. We found that removal of one copy of $d B R W D 3$ is sufficient to reduce the size and the frequency of hop ${ }^{\text {Tuml }}$-induced melanotic tumours (Fig. 3c; and see also Supplementary Table 3). Moreover, homozygous escapers of a putative hypomorphic allele of $d B R W D 3$, generated by excision of the original P-element, frequently develop ectopic wing vein material (Fig. 3d), a phenotype reminiscent of the weak loss-of-function stat92E $E^{H J}$ allele ${ }^{27}$. Taken together, these experiments suggest a role for $d B R W D 3$ in JAK/STAT signalling.

As a second example we analysed Ptp61F, a protein tyrosine phosphatase whose depletion led to an increase in JAK/STAT signalling activity. To perform an epistasis analysis we removed known pathway components and tested for the effect of simultaneously targeting $p t p 61 F$. Double RNAi against $p t p 61 F$ together with lacZ, dome or hop results in pathway stimulation (Fig. 4a). However, simultaneous removal of $p t p 61 F$ and stat $92 E$ is sufficient to prevent signalling (Fig. 4a). Loss of this phosphatase therefore results in the stimulation of Stat92E activity even in the absence of upstream components, indicating that Ptp61F negatively regulates the pathway downstream of JAK. We next asked whether Ptp61F also interferes with JAK/STAT signalling in vivo by using the $\mathrm{cg}$-Gal4 driver to misexpress $p t p 61 F$ in blood cells of hop ${ }^{\text {Tuml }}$ mutants. Misexpression of $p t p 61 F c$ in a hop ${ }^{T u m l}$ mutant background resulted in suppression of melanotic tumour formation, with the average frequency of large tumours reduced by approximately fourfold, an effect also observed after the misexpression of $d P I A S^{14}$ (Fig. 4b; see also Supplementary Table 3$)$. Alternative splicing of $p t p 61 F$ gives nuclear and cytoplasmic protein forms that both contain the same phosphatase domain ${ }^{28}$. However, the tumour suppressor phenotype is only observed with nuclear Ptp61Fc (Fig. 4c), an effect that is reproduced by overexpression in cell culture (Fig. 4c). These results are consistent with our identification of Ptp61F as a negative regulator of pathway activity and suggest that it may function by targeting phosphorylated, nuclear-localized Stat92E for deactivation.

Aberrant JAK/STAT signalling has been implicated in multiple human malignancies and its components have been proposed as molecular targets for the development of therapeutic compounds $s^{29,30}$. The genome-wide screen presented here has identified known and previously unknown genes and we have characterized their probable level of interaction using cell-based epistasis analysis. Of the 90 JAK/STAT modifiers identified, many have human homologues that remain to be characterized. We have performed an analysis of two examples in vivo and demonstrate their roles in regulating the pathway in Drosophila. One of these is a homologue of human BRWD3, a gene recently identified at the break point of a translocation isolated from multiple B-CLL patients ${ }^{3}$. Given our functional analysis of $d B R W D 3$ and the known roles for JAK/STAT signalling during normal haematopoiesis, it is possible that a breakdown in BRWD3-mediated STAT regulation may represent a molecular mechanism leading to the development of B-CLL. Thus, comprehensive genetic surveys by RNAi using Drosophila as a model organism represent a powerful approach for identifying targets relevant to human diseases.

\section{METHODS}

Constructs. The JAK/STAT firefly luciferase reporter $6 \times 2 \times$ DrafLuc was constructed by multimerization of a molecularly characterized Stat92E binding site present in the promoter of the endogenous target gene Draf ${ }^{\circ}$, whereas the $4 \times$ SocsLuc reporter is based on a single 290-base-pair (bp) region containing four potential Stat92E binding sites present within the first intron of $\operatorname{socs} 36 E$ (ref. 13) (see Supplementary Information for details). A Renilla luciferase reporter gene under the control of the constitutively active Actin $5 C$ promoter was co-transfected and used to monitor cell number (see Supplementary Information).

Genome-wide RNAi screening. A genome-wide RNAi library based on polymerase chain reaction (PCR) templates with an average length of $408 \mathrm{bp}$ (flanked by T7-promotor binding sites) was generated by in vitro transcription ${ }^{17}$ (see Supplementary Information). Primer and amplicon sequence information can be found at http://rnai.dkfz.de. For screening experiments, Drosophila $\mathrm{Kc}_{167}$ cells were maintained in Schneider's medium (Invitrogen) supplemented with 10\% fetal bovine serum (PAA) and $100 \mu \mathrm{g} \mathrm{ml}^{-1}$ penicillin-streptomycin (Invitrogen). A total of 57 384-well screening plates were loaded with approximately $75 \mathrm{nM}$ dsRNA in $5 \mu \mathrm{l}$ of $1 \mathrm{mM}$ Tris at $\mathrm{pH} 7 . \mathrm{Kc}_{167}$ cells were batch transfected with the appropriate reporters, pAct-Renilla and pAct-UpdGFP, and transferred to dsRNA-containing screening plates in serum-free medium after 7 h. For 384well plates, 15,000 cells in $20 \mu \mathrm{l}$ were dispensed per well using an automated liquid dispenser (MultiDrop, Thermo Labsystems) and incubated for $60 \mathrm{~min}$ before addition of serum-containing medium. After $5 \mathrm{~d}$, medium was removed, cells were lysed and both firefly and Renilla luciferase activities were determined (see also Supplementary Information for computational analysis).

Cell-based epistasis experiments. For epistasis experiments, cells were transfected with expression vectors to stimulate pathway activity (see below). After $7 \mathrm{~h}, 30,000$ cells in $50 \mu \mathrm{l}$ of serum-free medium were seeded into 96 -well plates (Greiner) containing the dsRNAs to be tested (listed in Fig. 2c). One hour later, $75 \mu \mathrm{l}$ medium supplemented with $10 \%$ fetal bovine serum was added to the cells. Cells were lysed after $5 \mathrm{~d}$ and luciferase activity measured.

Each dsRNA was tested for its ability to suppress pathway activity under three conditions: (1) in Upd-expressing cells (screening conditions); (2) in cells treated with Upd-conditioned medium (Upd-CM); and (3) in cells expressing hop ${ }^{\text {Tuml }}$. Specifically, for Upd overexpression $5 \times 10^{6} \mathrm{Kc}_{167}$ cells were transfected with $600 \mathrm{ng} p A c t-U p d G F P, 500 \mathrm{ng} 6 \times 2 \times$ DrafLuc reporter, $250 \mathrm{ng}$ pAc5.1-Sid$1,25 \mathrm{ng} p A c t-R L$ and $p A c 5.1$ to a total of $2 \mu \mathrm{g}$ DNA. For Hop ${ }^{\text {TumL }}$ overexpression, $5 \times 10^{6} \mathrm{Kc}_{167}$ cells were transfected with $200 \mathrm{ng}$ pAct-hop ${ }^{\text {TumL }}, 500 \mathrm{ng} 6 \times 2 \times$ DrafLuc reporter, $250 \mathrm{ng} p A c 5.1$-Sid-1, $25 \mathrm{ng} p A c t-R L$ and $p A c 5.1$ to a total of $2 \mu \mathrm{g}$ DNA. To analyse processes upstream of Upd, two batches of cells were transfected separately to generate 'responder' and 'Upd-producer' cells. The responder cells were batch transfected with $500 \mathrm{ng} 6 \times 2 \times$ DrafLuc reporter, $250 \mathrm{ng} p A c 5.1$-Sid-1, $25 \mathrm{ng} p A c t-R L$ and $p A c 5.1$ to a total of $2 \mu \mathrm{g}$ plasmid DNA and subsequently seeded into 96 -well plates containing the respective dsRNAs as described above. The Upd-producing cells were transfected with $2 \mu \mathrm{g}$ pActUpdGFP and cultured in $10 \mathrm{~cm}$ dishes (Falcon). After $4 \mathrm{~d}$, the cells were treated with $50 \mu \mathrm{g} \mathrm{ml}^{-1}$ heparin (Sigma) for $24 \mathrm{~h}$ and then the supernatant was collected, cleared by centrifugation and passed through a $0.2 \mu \mathrm{m}$ filter (Millipore). $50 \mu \mathrm{l}$ of this Upd-conditioned medium was then used to stimulate pathway activity in the responder cells for $24 \mathrm{~h}$. Control medium from untransfected heparin-treated cells did not elicit pathway activity (data not shown). Epistasis analysis of $p t p 61 F$ by double RNAi is described in Supplementary Information.

Genetics. For genetic interaction assays, females of the stock $y$, w, hop ${ }^{\text {Tuml }} / F M 7$; $P\left[w^{+}, c g\right.$-gal4.A $] 2$ (ref. 26) were crossed to wild-type controls (OreR and $w^{1118}$ ) and mutations in stat $92 E$ and $d B R W D 3$. The haemocyte specific Gal4 driver line $P\left[w^{+}, c g-G a l 4 . A\right] 2$ allowed specific misexpression constructs with upstream activating sequences (UAS) to be tested for their potential influence on tumour formation. Transgenic animals carrying UAS-EGFP or UAS- $\beta$-galactosidase were used as negative controls whereas UAS-dPIASGFP served as a positive control ${ }^{14}$ (see Supplementary Table 3).

Crosses were incubated at $25^{\circ} \mathrm{C}$ and adult females heterozygous for the hop ${ }^{\text {Tuml }}$ chromosome were scored within $24 \mathrm{~h}$ of eclosion for the presence of tumours, classified as small (one or two small melanotic spots as shown in Fig. 4b, right panel) or large (one or more large melanized growths or more than three small spots; Fig. 4b, left panel). Survival rates for hop ${ }^{\text {Tuml }}$ females seem to be independent of tumour frequency at the time point counted (data not shown). Assays were repeated at least twice for each genotype and a representative example from one experiment is shown (Fig. $4 \mathrm{~b}$ ).

Genetic interaction with $P\left[w^{+}, G M R-u p d \Delta 3^{\prime}\right]^{\prime} 19$ (termed GMR-upd $\Delta 3^{\prime}$ in the text) was undertaken as described ${ }^{25}$ using OreR and stat $92 E^{06346}$ as negative

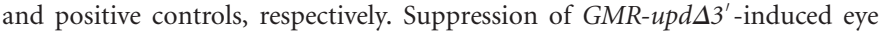
overgrowth by $A B R W D 3^{05842}$ was observed in multiple independent experiments in a majority of individuals of the appropriate genotype. Drosophila heads were photographed using a Zeiss STEMI 2000-C binocular microscope and Axiocam camera.

\section{Received 9 March; accepted 26 May 2005.}

1. Brivanlou, A. H. \& Darnell, J. E. Jr Signal transduction and the control of gene expression. Science 295, 813-818 (2002). 
2. Hombria, J. C. \& Brown, S. The fertile field of Drosophila Jak/STAT signalling. Curr. Biol. 12, R569-R575 (2002).

3. Kalla, C. et al. Translocation $t(X ; 11)(q 13 ; q 23)$ in B-cell chronic lymphocytic leukemia disrupts two novel genes. Genes Chromosom. Cancer 42, 128-143 (2005).

4. Binari, R. \& Perrimon, N. Stripe-specific regulation of pair-rule genes by hopscotch, a putative Jak family tyrosine kinase in Drosophila. Genes Dev. 8 300-312 (1994)

5. Harrison, D. A., McCoon, P. E., Binari, R., Gilman, M. \& Perrimon, N. Drosophila unpaired encodes a secreted protein that activates the JAK signalling pathway. Genes Dev. 12, 3252-3263 (1998)

6. Hou, X. S., Melnick, M. B. \& Perrimon, N. Marelle acts downstream of the Drosophila HOP/JAK kinase and encodes a protein similar to the mammalian STATs. Cell 84, 411-419 (1996).

7. Lagueux, M., Perrodou, E., Levashina, E. A., Capovilla, M. \& Hoffmann, J. A Constitutive expression of a complement-like protein in toll and JAK gain-offunction mutants of Drosophila. Proc. Natl Acad. Sci. USA 97, 11427-11432 (2000).

8. Boutros, M., Agaisse, H. \& Perrimon, N. Sequential activation of signalling pathways during innate immune responses in Drosophila. Dev. Cell 3, 711-722 (2002).

9. Meister, M. \& Lagueux, M. Drosophila blood cells. Cell. Microbiol. 5, 573-580 (2003).

10. Mukherjee, T., Castelli-Gair Hombría, J. \& Zeidler, M. P. Opposing roles for Drosophila JAK/STAT signalling during cellular proliferation. Oncogene 24, 2503-2511 (2005)

11. Brown, S., Hu, N. \& Castelli-Gair Hombria, J. Identification of the first invertebrate interleukin JAK/STAT receptor, the Drosophila gene domeless. Curr. Biol. 11, 1700-1705 (2001).

12. Yan, R., Small, S., Desplan, C., Dearolf, C. R. \& Darnell, J. E. Jr Identification of a Stat gene that functions in Drosophila development. Cell 84, 421-430 (1996).

13. Karsten, P., Hader, S. \& Zeidler, M. Cloning and expression of Drosophila SOCS36E and its potential regulation by the JAK/STAT pathway. Mech. Dev. 117, 343-346 (2002).

14. Betz, A., Lampen, N., Martinek, S., Young, M. W. \& Darnell, J. E. Jr A Drosophila PIAS homologue negatively regulates stat92E. Proc. Natl Acad. Sci. USA 98 9563-9568 (2001).

15. Mesilaty-Gross, S., Reich, A., Motro, B. \& Wides, R. The Drosophila STAM gene homolog is in a tight gene cluster, and its expression correlates to that of the adjacent gene ial. Gene 231, 173-186 (1999).

16. Kamath, R. S. et al. Systematic functional analysis of the Caenorhabditis elegans genome using RNAi. Nature 421, 231-237 (2003).

17. Boutros, M. et al. Genome-wide RNAi analysis of growth and viability in Drosophila cells. Science 303, 832-835 (2004).

18. Kittler, R. et al. An endoribonuclease-prepared siRNA screen in human cells identifies genes essential for cell division. Nature 432, 1036-1040 (2004).

19. Hannon, G. J. \& Rossi, J. J. Unlocking the potential of the human genome with RNA interference. Nature 431, 371-378 (2004)

20. Kwon, E. J. et al. Transcriptional regulation of the Drosophila raf proto-oncogene by Drosophila STAT during development and in immune response. J. Biol. Chem. 275, 19824-19830 (2000)

21. Clemens, J. C. et al. Use of double-stranded RNA interference in Drosophila cell lines to dissect signal transduction pathways. Proc. Natl Acad. Sci. USA 97, 6499-6503 (2000)

22. Sefton, L., Timmer, J. R., Zhang, Y., Beranger, F. \& Cline, T. W. An extracellular activator of the Drosophila JAK/STAT pathway is a sex-determination signal element. Nature 405, 970-973 (2000).

23. Luo, H., Hanratty, W. P. \& Dearolf, C. R. An amino acid substitution in the Drosophila hop ${ }^{\text {Tum-I }}$ Jak kinase causes leukemia-like hematopoietic defects. EMBO J. 14, 1412-1420 (1995).

24. Spradling, A. C. et al. The Berkeley Drosophila genome project gene disruption project: single P-element insertions mutating $25 \%$ of vital Drosophila genes. Genetics 153, 135-177 (1999).

25. Bach, E. A., Vincent, S., Zeidler, M. P. \& Perrimon, N. A sensitized genetic screen to identify novel regulators and components of the Drosophila janus kinase/signal transducer and activator of transcription pathway. Genetics 165, 1149-1166 (2003)

26. Harrison, D. A., Binari, R., Nahreini, T. S., Gilman, M. \& Perrimon, N. Activation of a Drosophila Janus kinase (JAK) causes hematopoietic neoplasia and developmental defects. EMBO J. 14, 2857-2865 (1995).

27. Yan, R., Luo, H., Darnell, J. E. Jr \& Dearolf, C. R. A JAK-STAT pathway regulates wing vein formation in Drosophila. Proc. Natl Acad. Sci. USA 93, 5842-5847 (1996)

28. McLaughlin, S. \& Dixon, J. E. Alternative splicing gives rise to a nuclear protein tyrosine phosphatase in Drosophila. J. Biol. Chem. 268, 6839-6842 (1993)

29. O'Shea, J. J., Pesu, M., Borie, D. C. \& Changelian, P. S. A new modality for immunosuppression: targeting the JAK/STAT pathway. Nature Rev. Drug Discov. 3, 555-564 (2004).

30. Levy, D. E. \& Darnell, J. E. Jr Stats: transcriptional control and biological impact. Nature Rev. Mol. Cell Biol. 3, 651-662 (2002).

Supplementary Information is linked to the online version of the paper at www.nature.com/nature.

Acknowledgements We are grateful to R. Paro for providing reagents to establish the genome-wide RNAi library. We wish to thank M. Yamayuchi and P. Karsten for the original $2 \times \operatorname{DrafSTAT}(w t)$ and Socs36E promoter plasmids, C. Hunter for the sid-1 plasmid, K. Bartscherer for advice and help with tissue culture experiments, T. Horn for bioinformatics support, and M. Stricker, B. Mosterman, I. Plischke and S. Häder for technical help. We thank H. Jäckle, S Cohen, M. Osborn, R. Paro and N. Pelte for comments on the manuscript. P.M. was supported by a fellowship from the German National Academic Foundation (Studienstiftung). Research in M.B. and M.P.Z. laboratories was supported in part by Emmy-Noether grants from the Deutsche Forschungsgemeinschaft.

Author Contributions This work has been a collaborative effort between the groups of M.B. and M.P.Z.; the Boutros laboratory contributing the functional genomic expertise to dissect signalling pathways and the Zeidler laboratory expertise working with JAK/STAT signalling.

Author Information Reprints and permissions information is available at npg.nature.com/reprintsandpermissions. The authors declare no competing financial interests. Correspondence and requests for materials should be addressed to M.B. (m.boutros@dkfz.de) or M.P.Z. (mzeidle@gwdg.de). 